\title{
KillerRed protein based in vivo photodynamic therapy and corresponding tumor metabolic imaging
}

\author{
Qiaoya $\operatorname{Lin}^{\dagger}$, Shuang Sha ${ }^{\dagger}$, Fei Yang, Honglin Jin and Zhihong Zhang* \\ Britton Chance Center for Biomedical Photonics \\ Wuhan National Laboratory for Optoelectronics \\ Huazhong University of Science and Technology \\ Wuhan 430074, P. R. China \\ MoE Key Laboratory for Biomedical Photonics \\ Department of Biomedical Engineering \\ Huazhong University of Science and Technology \\ Wuhan 430074, P. R. China \\ *czyzzh@mail.hust.edu.cn
}

Received 25 November 2015

Accepted 30 November 2015

Published 28 January 2016

\begin{abstract}
Photodynamic therapy (PDT) gains wide attention as a useful therapeutic method for cancer. It is mediated by the oxygen and photosensitizer under the specific light irradiation to produce the reactive oxygen species (ROS), which induce cellular toxicity and regulate the redox potential in tumor cells. Nowadays, genetic photosensitizers of low toxicity and easy production are required to be developed. KillerRed, a unique red fluorescent protein exhibiting excellent phototoxic properties, has the potential to act as a photosensitizer in the application of tumor PDT. Meantime, the course of tumor redox metabolism during this treatment was rarely investigated so far. Thus here, we investigated the effects of KillerRed-based PDT on tumor growth in vivo and examined the subsequent tumor metabolic states including the changes of nicotinamide adenine dinucleotide hydrogen $(\mathrm{NADH})$ and flavoprotein $(\mathrm{Fp})$, two important metabolic coenzymes of tumor cells. Results showed the tumor growth had been significantly inhibited by KillerRedbased PDT treatment compared to control groups. A home-made cryo-imaging redox scanner was used to measure intrinsic fluorescence and exogenous KillerRed fluorescence signals in tumors. The Fp signal was elevated by nearly 4.5 -fold, while the NADH signal decreased by $66 \%$ after light irradiation, indicating that Fp and NADH were oxidized in the course of KillerRedbased PDT. Furthermore, we also observed correlation between the fluorescence distribution of
\end{abstract}

* Corresponding author.

$\dagger$ These authors contributed equally to this work.

This is an Open Access article published by World Scientific Publishing Company. It is distributed under the terms of the Creative Commons Attribution 4.0 (CC-BY) License. Further distribution of this work is permitted, provided the original work is properly cited. 
KillerRed and NADH. It suggests that the KillerRed protein based PDT might provide a new approach for tumor therapy accompanied by altering tumor metabolism.

Keywords: Reactive oxygen species; redox; metabolism; flavoprotein; nicotinamide adenine dinucleotide hydrogen.

\section{Introduction}

PDT is a useful therapeutic method in the treatment of the head and neck, ${ }^{1}$ lung, brain, ${ }^{2}$ skin, breast $^{3}$ and pancreatic carcinomas in clinic. ${ }^{4}$ It is mediated by photosensitizer and oxygen under the specific light irradiation to produce the reactive oxygen species (ROS), ${ }^{5,6}$ which induce cellular toxicity and regulate the redox potential in tumor cells. $^{7,8}$ Recently, many researchers focus on the development of the photosensitizers to improve the efficacy of PDT method. ${ }^{9,10}$ Nowadays, most of photosensitizers are hydrophobic chemical materials and difficult to be cleared in bodies. Compared with the chemical photosensitizer, such as prophyrin related products, the genetically encoded protein has many advantages. For example, it is of low toxicity to organism and easy to obtain. Thus, developing a photosensitizer based on gene-encoded protein is of great value. ${ }^{11}$

KillerRed is a unique genetically encoded far red fluorescent protein mutated from the Hydrozoa jellyfish chromoprotein anm $2 \mathrm{CP}^{12}$ and has a phototoxicity 1000-fold more than that of green fluorescent protein (GFP). ${ }^{13}$ It has the potential to act as a photosensitizer. Some groups reported that this KillerRed protein could produce ROS which has toxicity to the tumor cells ${ }^{13,15}$ and its different subcellular component localization also had been found, such as membrane ${ }^{11,16}$ or mitochondria ${ }^{17}$ which play a major role in photodynamic therapy induced cell death. ${ }^{18-20}$ During the treatment of photodynamic therapy, the genetically encoded photosensitizer will alter the tumor cell redox state and metabolism, however, the course of tumor redox metabolism during PDT treatment was rarely investigated so far.

Tumor metabolism plays an important role in the tumor growth ${ }^{21}$ and differentiation, metastasis ${ }^{22}$ and apoptosis. ${ }^{23}$ The metabolism of tumor cells is different from most normal cells, which is called Warburg effect and is not an effective ATP generation way. ${ }^{24}$ Investigating tumor metabolism is critical because it can provide an open window for tumor therapeutics. ${ }^{25}$ There are two autofluorescent indicators, nicotinamide adenine dinucleotide hydrogen $(\mathrm{NADH})$ and flavoprotein $(\mathrm{Fp})$, as a redox pair to indicate the tumor redox metabolism state. ${ }^{26-29}$ They are both coenzymes in the mitochondrial respiration chain of all tissues. The fluorescence intensity ratio of $\mathrm{NADH} /(\mathrm{NADH}+\mathrm{Fp})$ can be as a standard for detecting tissue metabolism state which has been utilized for several years. ${ }^{30,31}$

In this paper, we investigated the antitumor efficacy of KillerRed protein-based PDT in human fibrocarcinoma bearing mice. Meanwhile, the tumor redox state was monitored during the treatment, including NADH and Fp signal collected in tumor cells. We found that the KillerRed protein based PDT inhibited the tumor growth and the tumor was oxidized during the PDT treatment evidenced by increased Fp signal and decreased NADH signal. Futhermore, we also investigated the relationship between exogenous KillerRed protein and NADH, and found that the fluorescence distribution of KillerRed and NADH had correlation evidenced by their high linear coefficient.

\section{Materials and Methods}

\subsection{Construction of plenti-hiko- KillerRed plasmid}

To obtain virus transfection plasmid, the virus plasmid vector of interest plenti-hiko-KillerRed was constructed as follows:

KillerRed-F: 5'AAAGGGTCTAGATAAGCAG AGCTGGTTTAGTGAACCGTCAGATCCGCT AG3';

KillerRed-R:5'CCCGGAATTCTTAATCCTC GTCGCTACCGATGGCGCTGGTGATGCGG3'

The primers were used to amplify the dmitoKillerRed gene. The amplification condition is: $96^{\circ} \mathrm{C}$ $3 \mathrm{~min}, 96^{\circ} \mathrm{C} 1 \mathrm{~min}, 60^{\circ} \mathrm{C} 1 \mathrm{~min}, 72^{\circ} \mathrm{C} 1.5 \mathrm{~min}, 40$ cycles, ended with $72^{\circ} \mathrm{C} 10 \mathrm{~min}$. After that, the KillerRed fragment and the vector plenti-hiko were digested by XbaI and EcoRI simultaneously and then ligated with T4 Ligase. 


\subsection{Virus packaging and cell infection}

After being constructed successfully, the plasmid was packaged with the third HIV lentivirus package system. $3 \times 10^{6}$ HEK-293T cells were seeded into three cell dishes, respectively, after the dishes were treated by the L-Polylysine (Sigma, USA) solution for $15 \mathrm{~min}$. When the HEK-293T cells were adherent, four plasmids (plenti-hiko-KillerRed, pMD2G, pRSV-REV and pMDLg-pRRE) were transfected into the cells simultaneously, $10 \mu \mathrm{g}$ for each plasmid, adding $2 \times \mathrm{HBS}(3.375 \mathrm{ml}), 2.5 \mathrm{M} \mathrm{CaCl}_{2}$ $(0.377 \mathrm{ml})$ and $\mathrm{ddH}_{2} \mathrm{O}(2.998 \mathrm{ml})$ respectively. Since $24 \mathrm{~h}$ post transfection, the supernatant of dishes were collected and replaced with fresh culture medium continually every other day for three days, and all supernatant was stored at $4^{\circ} \mathrm{C}$. The supernatant was filtered before the virus was concentrated by ultracentrifuge. HT1080 cells were seeded into 96 well-plate, $1 \times 10^{4}$ cells for each well. When the cells were adherent, the concentrated virus was added into the cultured HT1080 cells slowly. After $24 \mathrm{~h}$, the medium was replaced with fresh RPMI 1640 medium. The fluorescent HT1080 cells were digested into several 96 well-plates and the nonfluorescent cells were stamped by toothpick slowly.

\subsection{KillerRed-HT108O and HT1080 cells confocal imaging}

To observe the KillerRed protein and endogenous fluorescent images, $2 \times 10^{4}$ HT1080 cells and KillerRedHT1080 cells were seeded into the microscopy chamber, respectively. The KillerRed-HT1080 cells were irradiated by the $561 \mathrm{~nm}$ laser at the power of $100 \%$ for $30 \mathrm{~min}$ and then $1 \mu \mathrm{l}$ DCFH-DA (Beyotime, China) were added and incubated for $30 \mathrm{~min}$ before being washed away twice with PBS. The imaging was performed on a PE spinning disk confocal microscopy (Olympus, Japan). (The Fp fluorescent image was taken with a $520-540 \mathrm{~nm}$ emission filter excited by a $488 \mathrm{~nm}$ laser at $21 \%$ of the maximun power with an exposure time of $1 \mathrm{~s}$. The KillerRed fluorescent image was taken with a $582-700 \mathrm{~nm}$ emission filter excited by a $561 \mathrm{~nm}$ laser at $21 \%$ of the maximum power with an exposure time of $1 \mathrm{~s}$.)

\subsection{Cell culture and tumor treatment}

KillerRed-HT1080 cells were cultured in RPMI 1640 culture medium, (Gibico, USA) supplemented with
$10 \%$ fetal bovine serum (Gibico, USA) and $100 \mathrm{IU}$ penicillin, $100 \mathrm{mg} / \mathrm{ml}$ streptomycin at $37^{\circ} \mathrm{C} 5 \% \mathrm{CO}_{2}$. The cells were washed twice with PBS before being harvested with trypsin. Nude mice (male, $6-8$ weeks) were purchased from Silaike company (Shanghai, China). Mice were maintained under SPF conditions, and all animal experiments were performed according to the animal experiment guidelines of the Animal Experimentation Ethics Committee of HUST. Nude mice were inoculated with $2 \times 10^{6}$ KillerRed-HT1080 cells (in $100 \mu \mathrm{l}$ PBS) subcutaneously in the left flanks, respectively. Two weeks after that, before the tumor volume reached $1 \mathrm{~cm}^{3}$, mice were anesthetized with $2 \%(\mathrm{v} / \mathrm{v})$ isofluorane and irradiated once at the tumor area with a $561 \mathrm{~nm}$ laser (Coherent Asia, USA) at $80 \mathrm{mV}$ for $3 \mathrm{~min}$ every three days. The remaining normal tissue was protected by silver paper from the light irradiation.

\subsection{Redox imaging}

After being treated with irradiation, the tumor bearing mice were sacrificed immediately under anesthesia, The tumors were excised, and frozen in liquid nitrogen quickly before being mounted by refrigerant (ethanol: glycerin: water $=3: 6: 1$ ). In order to observe tumor metabolism directly and conveniently, we utilized a redox scanner, a homemade low temperature imaging system in our lab for monitoring tissue mitochondrial NADH and FAD fluorescent signal ex vivo. The fluorescent emission wavelength of NADH and Fp is $420-480 \mathrm{~nm}^{32}$ and $530-560 \mathrm{~nm}^{33}$ respectively. NADH fluorescence in tumors was excited by $360 \mathrm{~nm}$ LED (Edmund optics, USA), and collected using a $450 \mathrm{~nm}$ filter. The flavoprotein fluorescence was excited by the $450 \mathrm{~nm}$ LED, and the emission using $530 \mathrm{~nm}$ filter. KillerRed fluorescence was excited by $590 \mathrm{~nm}$ LED, and collected using $650 \mathrm{~nm}$ filter. The image data was analyzed by Matlab 7.0 software. The NADH and Fp redox images were performed following these formulas: Ratio ${ }_{\mathrm{PN}}=\mathrm{FL}_{\mathrm{NADH}} /\left(\mathrm{FL}_{\mathrm{NADH}}+\mathrm{FL}_{\mathrm{Fp}}\right)$ and Ratio $_{\mathrm{Fp}}=\mathrm{FL}_{\mathrm{Fp}} /\left(\mathrm{FL}_{\mathrm{NADH}}+\mathrm{FL}_{\mathrm{Fp}}\right)$.

\section{Results}

\subsection{Detection of ROS produced by KillerRed protein}

To investigate the antitumor efficacy of KillerRed protein based PDT in tumor bearing mice, we first 


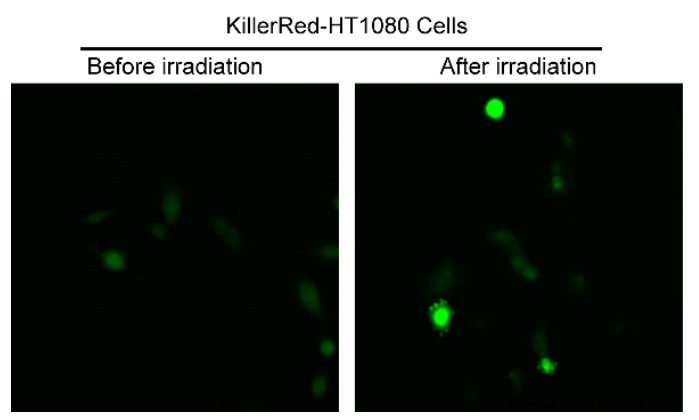

(a)

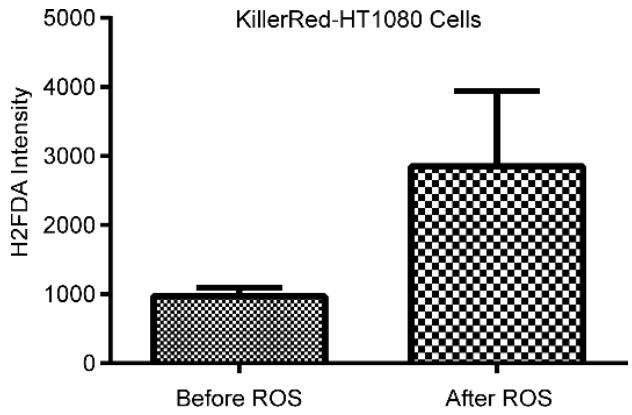

(b)

Fig. 1. The image of DCFH-DA stained the HT1080-KillerRed cells under the treatment of the light irradiation. (a) Left panel: The image of DCFH-DA stained the KillerRed-HT1080 cells with no irradiation. Right panel: DCFH-DA stained KillerRed-HT1080 cells under the irradiation for $30 \mathrm{~min}$ by $561 \mathrm{~nm}$ light. (b) Quantitative analysis from (a).

need to establish the cell line stably expressing KillerRed fluorescent protein. According to previously mentioned transfection method, we used lentivirus to infect the HT1080 cells. Fluorescence of dmito-KillerRed protein was detected in each cell, indicating that we successfully established a HT1080 cell line stably expressing KillerRed.

After that, in order to comfirm whether KillerRed protein in HT1080 cell produced ROS, the ROS detection experiment was performed. The fluorescent dye DCFH-DA was used to stain KillerRed-HT1080 cells, and imaged by confocal microscopy. As shown in Fig. 1(a), without irradiation, the fluorescence intensity of green dye DCFH-DA in KillerRed-HT1080 cells was low. After KillerRed-HT1080 cells were treated with the green light irradiation for $30 \mathrm{~min}$, the DCFH-DA dye fluorescence intensity in the KillerRed-HT1080 cells was enhanced [Fig. 1(a)]. Then the fluorescence intensity was quantified, showing that the green fluorescence intensity of DCFH-DA in the irradiated cells was three times stronger than the nonirradiated cells [Fig. 1(b)]. From the results we confirm that the KillerRed protein produced ROS under light irradiation. ${ }^{16}$

\subsection{Radiation raises cellular autofluorescence in KillerRed expression cells}

Since KillerRed protein could produce ROS in the course of green light irradiation, we next monitored the autofluorescence of cells during the irradiation with KillerRed protein. HT1080 cells and mitoKillerRed-HT1080 cells were irradiated with a $561 \mathrm{~nm}$ laser at $100 \%$ power at different time points $(0 \mathrm{~min}$, $15 \mathrm{~min}, 20 \mathrm{~min}$ and $30 \mathrm{~min}$ as indicated in Fig. 2), respectively. The cellular autofluorescent Fp signal was detected using a confocal imaging system [Fig. 2 (a)]. The Fp fluorescent signal through $515-550 \mathrm{~nm}$ was collected as the flavoprotein signal of cells. ${ }^{34-36}$

Fig. 2(a) showed Fp and KillerRed protein fluorescent images of KillerRed-HT1080 cells at different time points, the fluorescence intensity of $\mathrm{Fp}$ is 318.62 \pm 2.01 before irradiation, $397.75 \pm 13.67,395.46 \pm$ 14.26 and $394.04 \pm 14.52$ at $15 \mathrm{~min}, 20 \mathrm{~min}, 30 \mathrm{~min}$ post-irradiation, respectively [Fig. 2(b)]. The data indicated Fp fluorescent signal increased after irradiation. In the meantime, the KillerRed fluorescence was photobleached completely after light irradiation for $15 \mathrm{~min}$. In contrast, no significant difference was found between different time points post-irradiation in control HT1080 cells. As well known, when flavoprotein is oxidized, its fluorescent signal will increase. So we can conclude that the KillerRed fluorescent protein under the light irradiation can induce flavoprotein oxidization in cells in vitro.

\subsection{In vivo antitumor efficacy by KillerRed protein based PDT}

Since KillerRed protein under light irradiation could produce ROS efficiently in vitro, its antitumor efficacy in tumor bearing mice based photodynamic therapy needs further investigation in vivo. Three groups were used in this study: KillerRed-HT1080 with irradiation, KillerRed-HT1080 without irradiation and HT1080 with irradiation. The tumor bearing mice were treated with light irradiation once every three days. The treatment started at 8 days posttumor inoculation, when the tumor diameter is about $3.5 \mathrm{~mm}$. Meanwhile, the tumor volumes were monitored during the whole treatment. As shown in Fig. 3, 


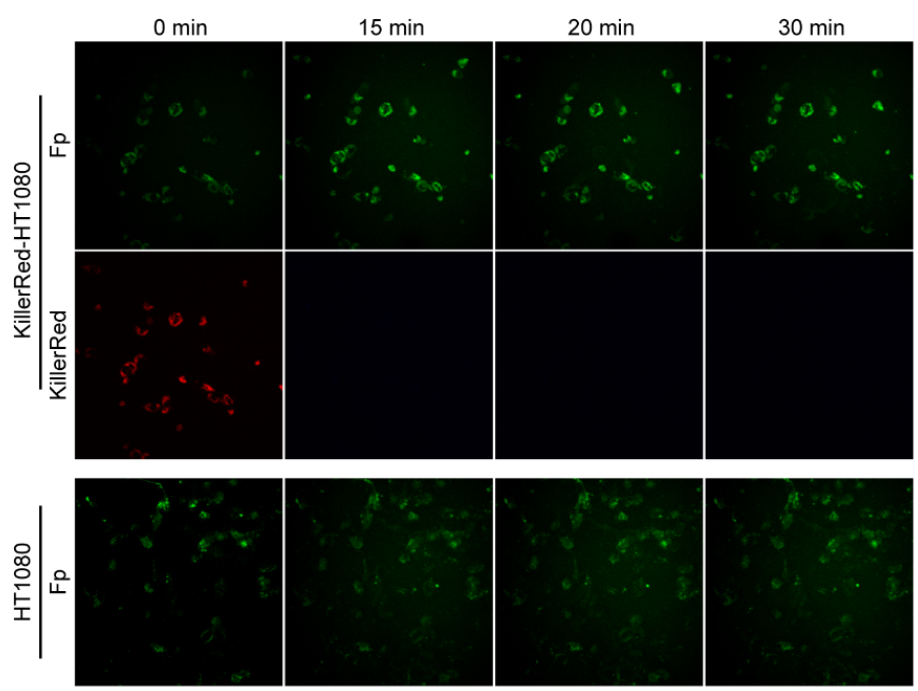

(a)

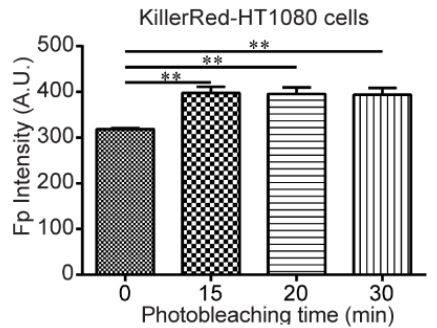

(b)

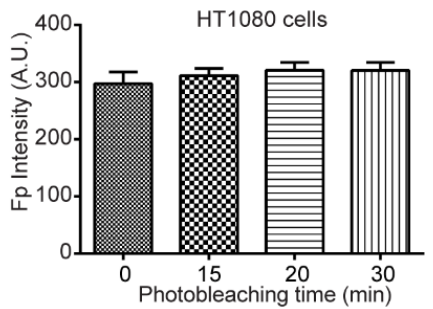

(c)

Fig. 2. Autofluorescence and KillerRed fluorescence after green light irradiation. (a) The flavoprotein fluorescence intensities of HT1080 cells and KillerRed-HT1080 cells under the irradiation by $561 \mathrm{~nm}$ laser for $0 \mathrm{~min}, 15 \mathrm{~min}, 20 \mathrm{~min}$, and $30 \mathrm{~min}$. The first row: the Fp images of KillerRed-HT1080 cells. The middle row: the KillerRed fluorescent images of KillerRed-HT1080 cells. The third row: the Fp images of the HT1080 cells. (b) The quantitative fluorescence intensity analysis of KillerRed-HT1080 cells. (c) The quantitative fluorescence intensity analysis of HT1080 cells. The scale bars represent Mean \pm SEM. $(* * p<0.01)$.

the tumor growth of KillerRed-HT1080 tumor bearing mice under laser irradiation had been significantly inhibited compared to the other control groups. While there was no significant difference between KillerRed-HT1080 tumors without irradiation and HT1080 tumors with irradiation. From the results, we can conclude that the KillerRed protein based photodynamic therapy could efficiently inhibit the

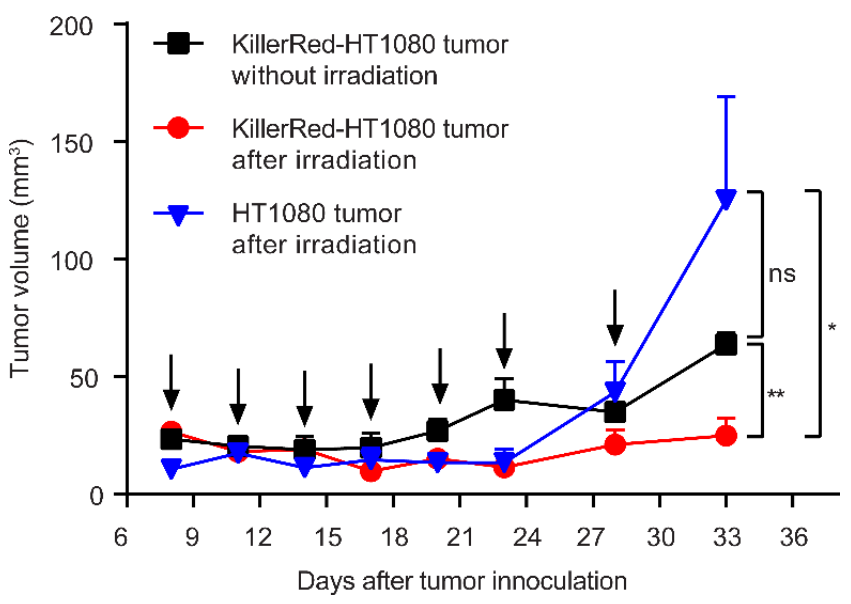

Fig. 3. The tumor volume measurement of the KillerRedHT1080 tumors and HT1080 tumors. The black arrows indicate the time points at which the mice were treated with light irradiation. The scale bars represent Mean \pm SEM. $\left({ }^{*} p<0.05\right.$, $\left.{ }^{* *} p<0.01, n=4\right)$. human fibrocarcinoma tumor growth and it suggests KillerRed can be utilized as a photosensitizer in vivo.

\subsection{Cyro-imaging of KillerRed- HT1080 tumors}

In order to know the tumor metabolism effect of KillerRed during the course of photodynamic therapy, the cryo-images of KillerRed-HT1080 tumor and HT1080 tumor were collected to monitor the metabolism during PDT treatment. The corresponding NADH, Fp, Ratio ${ }_{\mathrm{NADH}}$ and Ratio Fp $_{\text {of }}$ tumors are shown in the Fig. 4 . The tumors were frozen in liquid nitrogen after a $561 \mathrm{~nm}$ laser irradiation $(80 \mathrm{~mW})$ for three minutes and imaged using cryo-imaging system. The metabolism images of tumor samples at different depth levels $(500 \mu \mathrm{m}$, $1000 \mu \mathrm{m}, 1500 \mu \mathrm{m})$ were taken. The white circle region of Fp images in Fig. 4(a) (1000 $\mu \mathrm{m})$ indicates the irradiation region inside tumors. The KillerRed protein in that region was photobleached with the fluorescent signal disappeared. The corresponding NADH fluorescence intensity was decreased and Fp fluorescence intensity was increased obviously at the irradiation region compared to no irradiation region (Fig. 4). The figure below is the histogram of the corresponding fluorescence intensity. The 


\section{Q. Lin et al.}

images taken at other levels (images not shown here) were consistent with images shown in Fig. 4 (a). The fluorescence intensity of Fp and $\mathrm{NADH}$, and corresponding Ratio ${ }_{\mathrm{NADH}}$ and Ratio ${ }_{\mathrm{Fp}}$ in both groups at the depth of $500 \mu \mathrm{m}, 1000 \mu \mathrm{m}, 1500 \mu \mathrm{m}$ were quantified respectively [Figs. 4(c)-4(f)]. It showed that the $\mathrm{NADH}$ intensity after irradiation decreased by nearly
$66 \%$ in average at different section level compared to nonirradiation group [Fig. 4(c)], while the Fp intensity increased nearly 4.5 folds [Fig. 4(d)]. Correspondingly, the Ratio ${ }_{\mathrm{NADH}}$ after irradiation decreased by nearly $72 \%$, while Ratio ${ }_{F p}$ increased by 3.8 folds [Figs. 4(e) and 4(f)]. The data indicated that the tumor metabolism was changed under light
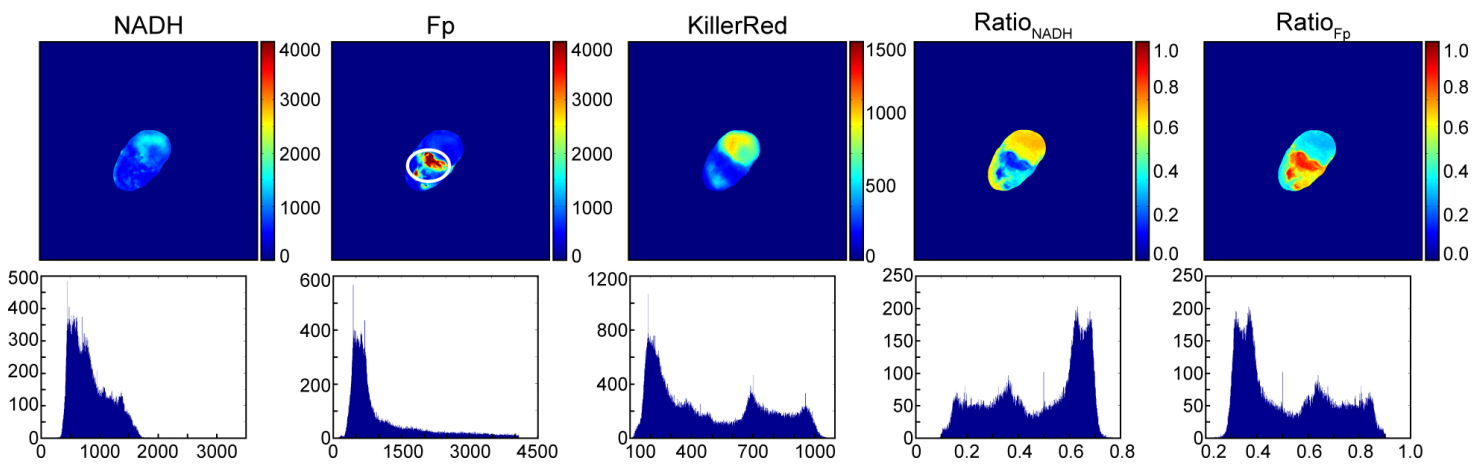

(a)
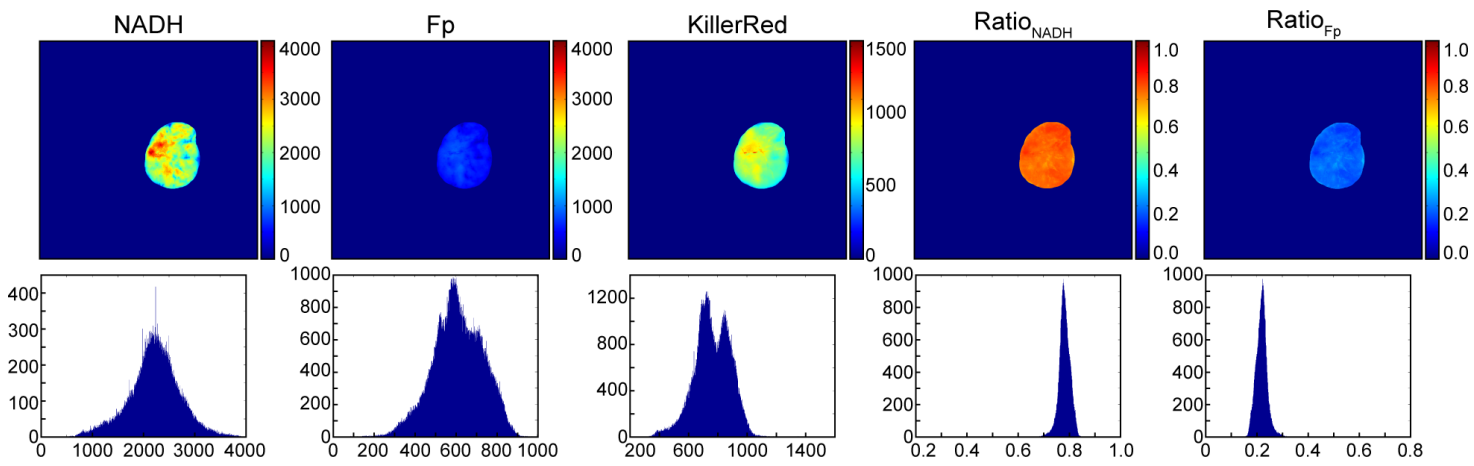

(b)

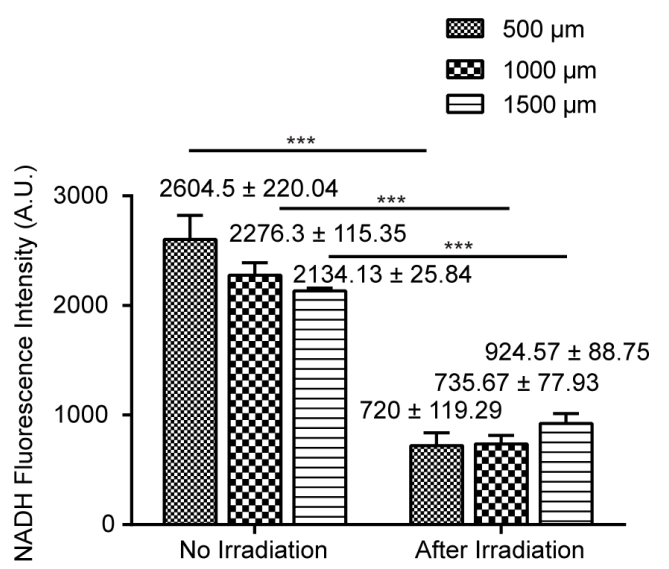

(c)

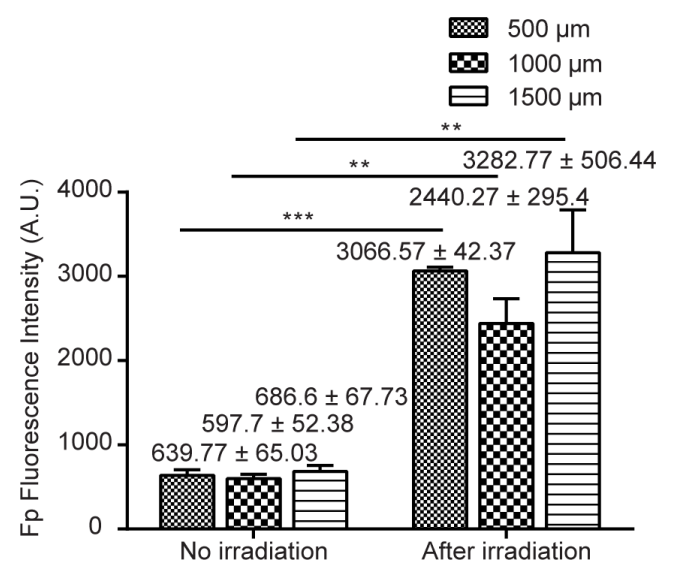

(d)

Fig. 4. Cyro-imaging of KillerRed-HT1080 tumor by light irradiation and no irradiation. (a) The cyro-imaging of KillerRedHT1080 tumor treated by light irradiation. (b) The cyro-imaging of KillerRed-HT1080 tumor with no irradiation. (c)-(f) The quantification data on fluorescence intensity of Fp at different depth, including $500 \mu \mathrm{m}, 1000 \mu \mathrm{m}, 1500 \mu \mathrm{m}$. 


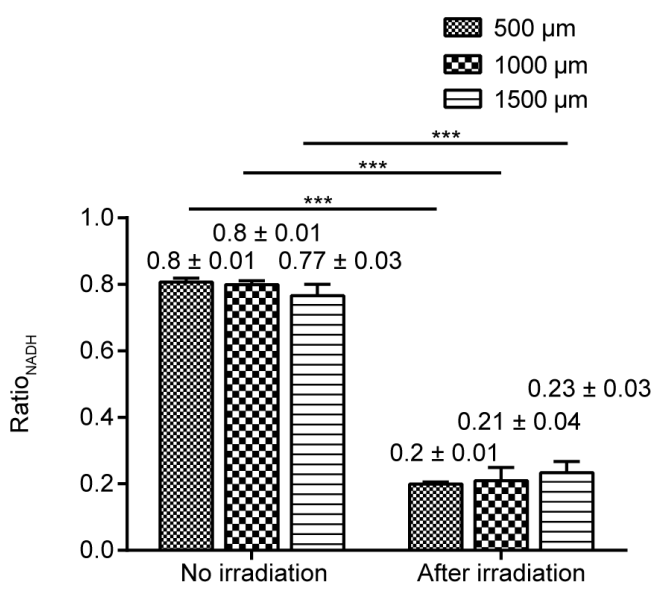

(e)

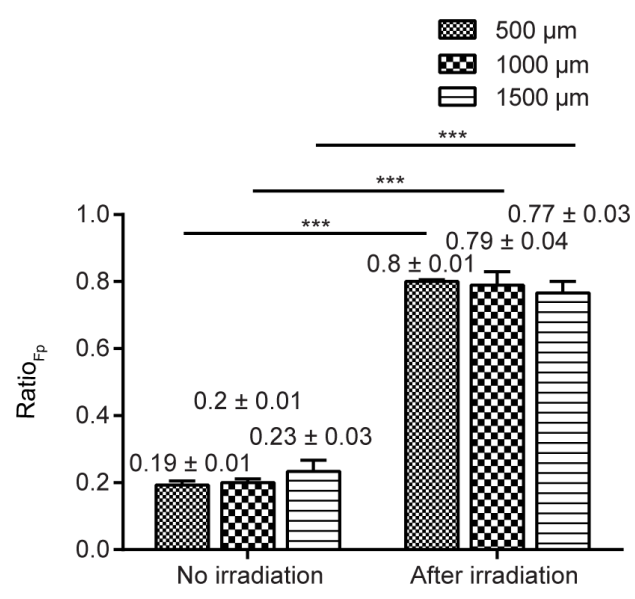

(f)

Fig. 4. (Continued)

irradiation in photodynamic therapy while oxidizing the NADH and Fp simultaneously.

\subsection{The correlation between KillerRed protein and NADH signal}

We also observed that the distribution of KillerRed fluorescent signal in tumors is similar with that of
NADH signal both in irradiation group and nonirradiation group, for which the correlation analysis was performed. The result showed that the coefficient of determination $R^{2}$ in KillerRed-HT1080 tumor was 0.859 in irradiation group, and 0.701 in nonirradiation group. High coefficient value $(>0.5)$ indicated that the distribution of KillerRed had correlation with that of NADH. It suggested that
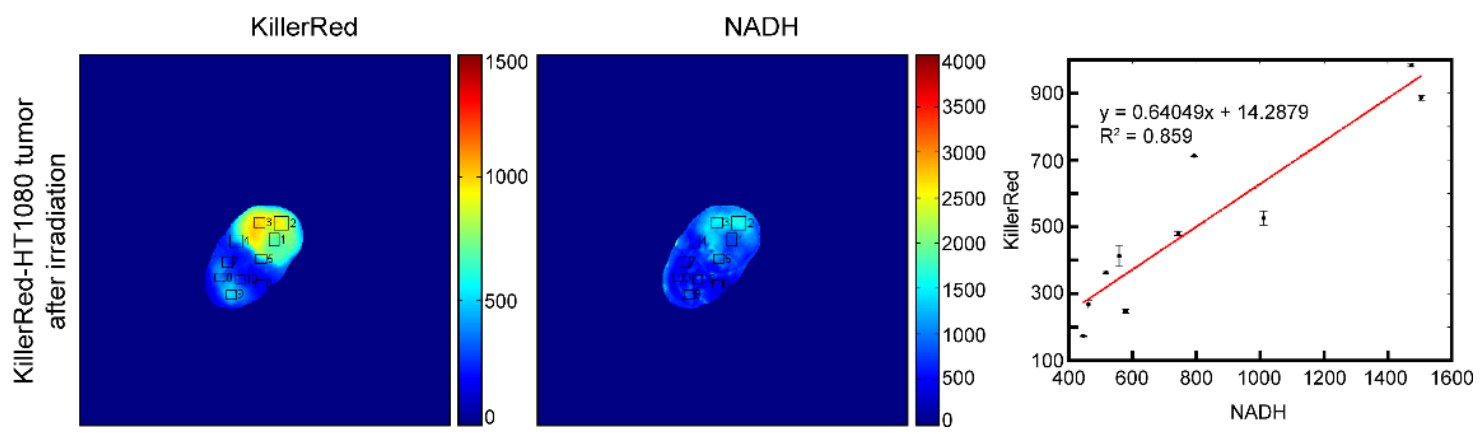

(a)
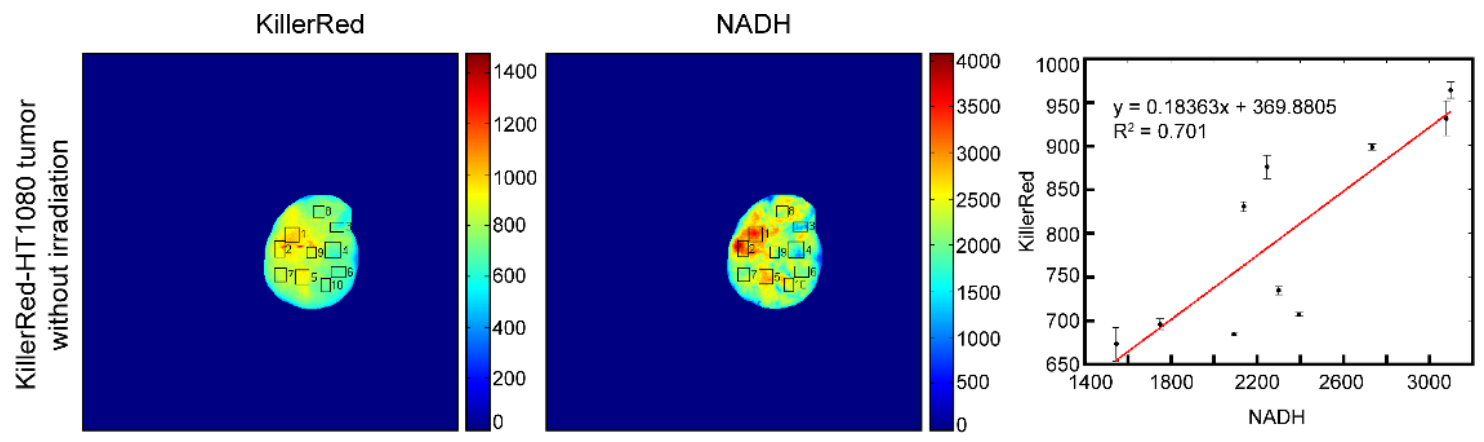

(b)

Fig. 5. The correlation between the distribution of KillerRed protein and that of NADH. (a) Tumors after irradiation treatment and (b) Tumors without irradiation. 
the tumor redox state, especially the reduction state, had a relationship with the exogenous protein expression.

\section{Discussion and Conclusion}

KillerRed protein is a potential genetically encoded photosensitizer which can produce ROS by chromophore-assisted light inactivation (CALI) under light irradiation. ${ }^{14}$ The ROS accumulated in tumor cells will induce tumor cells apoptosis. Previous study considered that the KillerRed protein can be used as a potential photosensitizer in tumor photodynamic therapy, ${ }^{5}$ however, rarely reports have investigated its antitumor effect in vivo. Moreover, the effect of photodynamic therapy on the tumor metabolism is not very clear either. In this study, we investigated the effect on the metabolism of tumor cells in vitro and in vivo by KillerRed protein when using it as a photosensitizer in a xenograft tumor model.

In our study, it was demonstrated that the KillerRed protein could efficiently produce ROS in vitro (Fig. 1). We irradiated KillerRed-HT1080 cells via $561 \mathrm{~nm}$ laser at different time points, and detected the endogenous fluorescence intensity. The results showed that the endogenous Fp fluorescence intensity in cells raised obviously compared to HT1080 cells after irradiation for $15 \mathrm{~min}$ (Fig. 2). Moreover, the increased Fp signal was maintained at least to $30 \mathrm{~min}$, demonstrating that the flavoprotein was irreversibly oxidized. When utilizing KillerRed protein as a photosensitizer to treat human fibrosarcoma with photodynamic therapy, we observed that the tumor growth was efficiently inhibited (Fig. 3). Under light irradiation, KillerRed protein in tumor region produced ROS, which efficiently killed tumor cells in vivo. In the meantime, the tumor endogenous NADH and Fp were oxidized (Fig. 4). It suggests that the KillerRed protein could be used as an efficient photosensitizer in the application of tumor therapy in future.

With tumor progression, the redox metabolism is variable and complicated. Whether the tumor redox state will affect the exogenous gene expression or not is still not clear now. In our study, we also demonstrated that the exogenous protein expression of KillerRed in tumor had relationship with the tumor redox state of NADH. We hypothesized this phenomenon may be induced by KillerRed protein maturation process which requires oxygen, ${ }^{37}$ since $\mathrm{NADH}$ is a common reduced state indicator.
In summary, this study demonstrated the antitumor efficacy of KillerRed protein as an alternative PDT photosensitizer and provided useful information regarding the tumor metabolic states following KillerRed-based PDT. It also suggests that KillerRed protein based PDT has broad application for tumor therapy accompanied by altering tumor metabolism in future.

\section{Acknowledgment}

We thank the Optical Bioimaging Core Facility of WNLO-HUST for the support in data acquisition, and the Analytical and Testing Center of HUST for spectral measurements. We thank Prof. Zhenli Huang in our lab for providing the solid-state laser for us. This work was supported by the Major Research plan of the National Natural Science Foundation of China (Grant No. 91442201), the China Postdoctoral Science Foundation funded project (Grant No. 2015M572148, 2012M521430 and 2013T60721) and the Open Research Fund of State Key Laboratory of Bioelectronics, Southeast University.

\section{References}

1. M. A. Biel, "Photodynamic therapy and the treatment of head and neck neoplasia," The Laryngoscope 108, 1259-1268 (1998).

2. M. A. Rosenthal, B. Kavar, J. S. Hill, D. J. Morgan, R. L. Nation, S. S. Stylli, R. L. Basser, S. Uren, H. Geldard, M. D. Green, S. B. Kahl, A. H. Kaye, "Phase I and pharmacokinetic study of photodynamic therapy for high-grade gliomas using a novel boronated porphyrin," J. Clin. Oncol. 19, 519-524 (2001).

3. A. Dimofte, T. C. Zhu, S. M. Hahn, R. A. Lustig, "In vivo light dosimetry for motexafin lutetiummediated PDT of recurrent breast cancer," Lasers Surg. Med. 31, 305-312 (2002).

4. D. E. Dolmans, D. Fukumura, R. K. Jain, "Photodynamic therapy for cancer," Nat. Rev. Cancer 3, 380-387 (2003).

5. M. V. Shirmanova, E. O. Serebrovskaya, K. A. Lukyanov, L. B. Snopova, M. A. Sirotkina, N. N. Prodanetz, M. L. Bugrova, E. A. Minakova, I. V. Turchin, V. A. Kamensky, S. A. Lukyanov, E. V. Zagaynova, "Phototoxic effects of fluorescent protein KillerRed on tumor cells in mice," J. Biophotonics 6, 283-290 (2013).

6. K. M. S. Russell, B. Vegh, Marina K. Kuimova, "Reactive oxygen species in photochemistry of the 
red fluorescent protein "Killer Red," Chem. Commun. 4, 4887-4889 (2011).

7. V. Adler, Z. Yin, K. D. Tew, Z. Ronai, "Role of redox potential and reactive oxygen species in stress signaling," Oncogene 18, 6104-6111 (1999).

8. V. Irihimovitch, M. Shapira, "Glutathione redox potential modulated by reactive oxygen species regulates translation of Rubisco large subunit in the chloroplast," J. Biol. Chem. 275, 16289-16295 (2000).

9. Y. Ye, L. X. Wang, D. P. Zhang, Y. J. Yan, Z. L. Chen, "Studies on photodynamic mechanism of a novel chlorine derivative (TDPC) and its antitumor effect for photodynamic therapy in vitro and in vivo," J. Innov. Opt. Health. Sci. 8, (2015).

10. R. W. K. Wu, E. S. M. Chu, Z. Huang, M. C. Olivo, D. C. W. Ip, C. M. N. Yow, "An in vitro investigation of photodynamic efficacy of FosPeg (R) on human colon cancer cells," J. Innov. Opt. Health. Sci. 8, (2015).

11. Z. X. Liao, Y. C. Li, H. M. Lu, H. W. Sung, "A genetically-encoded KillerRed protein as an intrinsically generated photosensitizer for photodynamic therapy," Biomaterials 35, 500-508 (2014).

12. G. Mueller, "From green to red - To more dead? Autofluorescent proteins as photosensitizers," $J$. Photoch. Photobio. B 9, 95-98 (2010).

13. M. E. Bulina, K. A. Lukyanov, O. V. Britanova, D. Onichtchouk, S. Lukyanov, D. M. Chudakov, "Chromophore-assisted light inactivation (CALI) using the phototoxic fluorescent protein KillerRed," Nat. Protoc. 1, 947-953 (2006).

14. M. E. Bulina, D. M. Chudakov, O. V. Britanova, Y. G. Yanushevich, D. B. Staroverov, T. V. Chepurnykh, E. M. Merzlyak, M. A. Shkrob, S. Lukyanov, K. A. Lukyanov, "A genetically encoded photosensitizer," Nat. Biotechnol. 24, 95-99 (2006).

15. E. O. Serebrovskaya, E. F. Edelweiss, O. A. Stremovskiy, K. A. Lukyanov, D. M. Chudakov, S. M. Deyev, "Targeting cancer cells by using an antireceptor antibody-photosensitizer fusion protein," Proc. Natl. Acad. Sci. USA 106, 9221-9225 (2009).

16. W. Waldeck, G. Mueller, M. Wiessler, K. Toth, K. Braun, "Positioning effects of KillerRed inside of cells correlate with DNA strand breaks after activation with visible light," Int. J. Med. Sci. 8, 97-105 (2011).

17. T. Shibuya, Y. Tsujimoto, "Deleterious effects of mitochondrial ROS generated by KillerRed photodynamic action in human cell lines and C. elegans," J. Photoch. Photobio. 117, 1-12 (2012).

18. J. Morgan, A. R. Oseroff, "Mitochondria-based photodynamic anti-cancer therapy," Adv. Drug. Deliv. Rev. 49, 71-86 (2001).
19. X. Wang, "The expanding role of mitochondria in apoptosis," Genes. Dev. 15, 2922-2933 (2001).

20. S. Orrenius, "Mitochondrial regulation of apoptotic cell death," Toxicol. Lett. 149, 19-23 (2004).

21. M. Ristow, "Oxidative metabolism in cancer growth," Curr. Opin. Clin. Nutr. 9, 339-345 (2006).

22. L. Z. Li, "Imaging mitochondrial redox potential and its possible link to tumor metastatic potential," J. Bioenerg. Biomembr. 44, 645-653 (2012).

23. J. W. Locasale, L. C. Cantley, "Metabolic flux and the regulation of mammalian cell growth," Cell Metab. 14, 443-451 (2011).

24. P. P. Hsu, D. M. Sabatini, "Cancer cell metabolism: Warburg and beyond," Cell 134, 703-707 (2008).

25. M. G. Vander Heiden, "Targeting cancer metabolism: A therapeutic window opens," Nat. Rev. Drug Discov. 10, 671-684 (2011).

26. P. G. Michal Mokry, B. Vidinsky, "in vivo monitoring the changes of interstitial $\mathrm{pH}$ and FAD/ NADH," Photochem. Photobiol. 8, 793-797 (2006).

27. Z. Zhang, H. Li, Q. Liu, L. Zhou, M. Zhang, Q. Luo, J. Glickson, B. Chance, G. Zheng, "Metabolic imaging of tumors using intrinsic and extrinsic fluorescent markers," Biosens. Bioelectron. 20, 643-650 (2004).

28. B. Chance, B. Schoener, R. Oshino, F. Itshak, Y. Nakase, "Oxidation-reduction ratio studies of mitochondria in freeze-trapped samples. NADH and flavoprotein fluorescence signals," J. Biol. Chem. 254, 4764-4771 (1979).

29. L. Z. Li, R. Zhou, H. N. Xu, L. Moon, T. Zhong, E. J. Kim, H. Qiao, R. Reddy, D. Leeper, B. Chance, J. D. Glickson, "Quantitative magnetic resonance and optical imaging biomarkers of melanoma metastatic potential," Proc. Natl. Acad. Sci. USA 106, 6608-6613 (2009).

30. K. P. Q. Irene Georgakoudi, "Optical imaging using endogenous contrast to assess metabolic state," Annu. Rev. Biomed. Eng. 14, 351-367 (2012).

31. D. B. Zhihong Zhang, Hui Li, "Redox ratio of mitochondria as an indicator for the response of photodynamic therapy, "J. Biomed. Opt. 9, 772-778 (2004).

32. A. Mayevsky, G. G. Rogatsky, "Mitochondrial function in vivo evaluated by $\mathrm{NADH}$ fluorescence: from animal models to human studies," Am. J. Physiol Cell Ph. 292, C615-C640 (2007).

33. K. D. V. Jayanth Kumar, Ganesan Singaravelu, "In vivo estimation of redox states with autofluorescence spectroscopy in oral submucous fibrosis patients: A Pilot Study," J. Indian Acad. Oral Med. Radiol. 24, 257-260 (2012).

34. M. S. Islam, M. Honma, T. Nakabayashi, M. Kinjo, $\mathrm{N}$. Ohta, "pH dependence of the fluorescence 
Q. Lin et al.

lifetime of FAD in solution and in cells," Int. J. Mol. Sci. 14, 1952-1963 (2013).

35. J. V. Rocheleau, W. S. Head, D. W. Piston, "Quantitative NAD $(\mathrm{P}) \mathrm{H} /$ flavoprotein autofluorescence imaging reveals metabolic mechanisms of pancreatic islet pyruvate response," J. Biol. Chem. 279, 31780-31787 (2004).
36. A. V. Kuznetsov, J. Troppmair, R. Sucher, M. Hermann, V. Saks, R. Margreiter, "Mitochondrial subpopulations and heterogeneity revealed by confocal imaging: Possible physiological role?" Biochim. Biophys. Acta. 1757, 686-691 (2006).

37. R. Y. Tsien, "The green fluorescent protein," Annu. Rev. Biochem. 67, 509-544 (1998). 